\title{
Horizontal projectile motion: comparing free fall and drag resistance
}

\author{
R. Yáñez Valdez, P. A. Gómez Valdez, and F. de Armas Rivero \\ División de Ingeniería Mecánica e Industrial, Facultad de Ingeniería, Universidad Nacional Autónoma de México. \\ Circuito exterior s/n, Ciudad Universitaria, 07510, CDMX, México.
}

Received 4 February 2020; accepted 26 March 2020

\begin{abstract}
The motion of a particle that is projected into a resistant medium and subjected to a uniform gravitational field is considered. The drag force that acts upon the particle within the medium is proportional to the particle's speed, the density of the medium, and the cross-section area of the projectile. We review the problem of a horizontal motion with a drag force that is linear in speed. The problem is formulated in terms of particle speed, mass, height, time, and expelled gas velocity. The equations of motion are solved analytically, and a case study is discussed. As a result, we obtain the deviation of the projectile as a function of time because of the expelled gases with or without drag force.

Keywords: Newtons Laws; drag force; projectile.

El movimiento de una partícula que es proyectada en un medio con fricción y sujeta a un campo gravitacional uniforme es considerada. La fuerza de arrastre que actua sobre la partícula debida al medio es proporcional a la velocidad de la partícula, la densidad del medio y la sección transversal del proyectil. En este trabajo se revisa el problema del movimiento horizontal con fuerza de arrastre lineal en velocidad. Dicho problema es formulado en terminos de la velocidad de la partícula, la masa, la altura, el tiempo y la velocidad del gas expulsado. Las ecuaciones de movimiento son resueltas de manera analítica, y un caso de estudio es discutido. Como resultado, se obtiene la desviación del proyectil como función del tiempo debida a la expulsión del gas con o sin fuerza de arrastre.
\end{abstract}

Descriptores: Leyes de Newton; fuerza de arrastre; proyectil.

PACS: $01.40 . \mathrm{Ha} ; 45.20 . \mathrm{D}$

DOI: https://doi.org/10.31349/RevMexFisE.17.156

\section{Introduction}

Newton's second law was stated in the masterpiece of Sir Isaac Newton, "Philosophiae Naturalis Principia Mathematica", in 1687 [1].

This law establishes a quantification method for the concept of force and describes that the acceleration of a body is proportional to the force applied on it and is inversely proportional to its mass, i.e.,

$$
\sum \mathbf{F}=m \mathbf{a}
$$

That means that the heavier the body is, the stronger the applied force must be in order to achieve greater acceleration. This relation is true when the mass of the body is constant, but what happens if the mass is not constant? A good example of this is a rocket system that burns fuel as it rises in the air; in this case, Eq. (1) is not correct. Now, remembering that Newton stated his law as

$$
\sum \mathbf{F}=\frac{d(m \mathbf{v})}{d t}
$$

In this way it is possible to relate force with mass and acceleration. In the case where the mass is constant, it can be pulled out of the derivate and thus we obtain Eq. (1); therefore, the force is a concept that is equal to the derivate of the linear momentum of a given particle. This law helps us to understand why it is harder to accelerate a heavier object than a lighter one.

Rocket propulsion is an interesting field of study because its own method of acceleration is different from that of any other object. Compared to a runner, who uses the ground to accelerate by pushing against the ground constantly with his feet, or a car, which uses the road to accelerate due to the friction between the wheels and the road surface, or even a plane, which uses chemical energy from the fuel for heating and accelerating the air that is expelled from the rear side of a turbine, a rocket moves itself by expelling its own mass rearward. Thus, a rocket does not need a medium to impulse itself. If an object does not have any external agent that pushes it or one that it can push, how can the rocket get moving? The engine of a rocket is designed to throw the burned fuel out rearward, and by Newton's third law, the fuel pushes the rocket forward. If the reader has ever tried to build a prototype and recreate the experience of launching a scale rocket, they could have had some questions, e.g., what is the influence of air resistance? After obtaining the equations for the velocity as a function of time of a rocket fired vertically upward near the surface of the Earth, what are the conditions for liftoff? If the rocket's initial motion followed a horizontal path instead of a vertical one, the same questions would apply.

An object moving through a fluid is influenced by the resistant force, or drag, that acts oppositely to the relative motion of objects. Depending upon the characteristics of the flow, represented by a Reynolds number, two different types of drag models are used: a linear drag model and a quadratic drag model. To handle a projectile's motion while incorporating these drag models, various researches have been conducted to find their solutions while addressing several aspects of the problem [2-7]. Since neglecting the resistant force to projectile motion is impractical, is desirable to develop analytic solutions from a simplified model so as to elucidate 
some of the essential features and structure of this problem. The linear model, in which the resistance is taken to be proportional to the instantaneous velocity of the projectile, is generally accepted as the first approximation to such resistive behavior $[8,9]$ and it is the model we use here. Simulation of the projectile's motion with and without drag forces is extended. The equation of motion was formulated in order to cancel the inertial velocity of the projectile mounted on a mobile that is able to move at a constant inertial velocity.

\section{Variable mass problem}

Rocket propulsion is based on the principle that the rate of change of the linear momentum is equivalent to an external net force, and if the external net force is zero, the change in the linear momentum is also zero and so the linear momentum must be conserved. Consider Fig. 1, in which a rocket of mass $m$ is shown to be under the action of an external force $\mathbf{F}$, is expelling gas with a constant velocity $\mathbf{u}$ relative to the rocket, and has a time-dependent velocity $\mathbf{v}$.

Let the initial linear momentum of the rocket be written as

$$
\mathbf{p}_{i}=m \mathbf{v}
$$

As soon as the rocket begins to burn and expel gas at velocity $u$ relative to it, its mass decreases as a function of time. Let

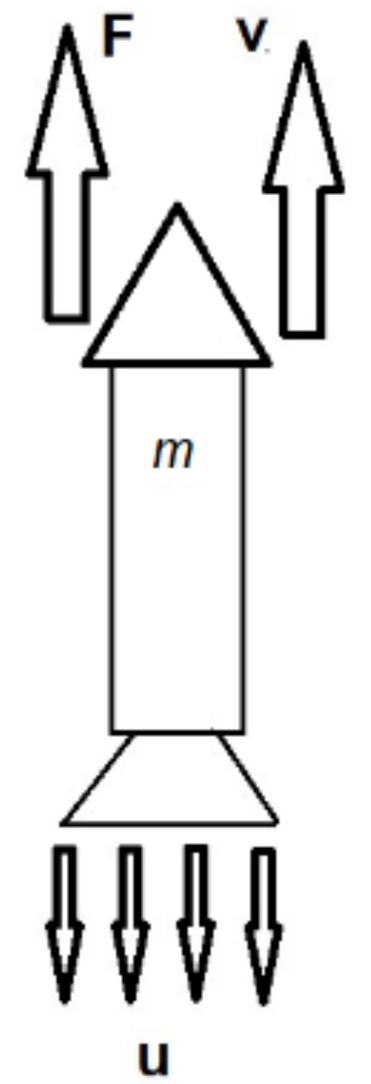

FIGURE 1. Mass rocket $m$ under the action of an external force $\mathbf{F}$ [10].

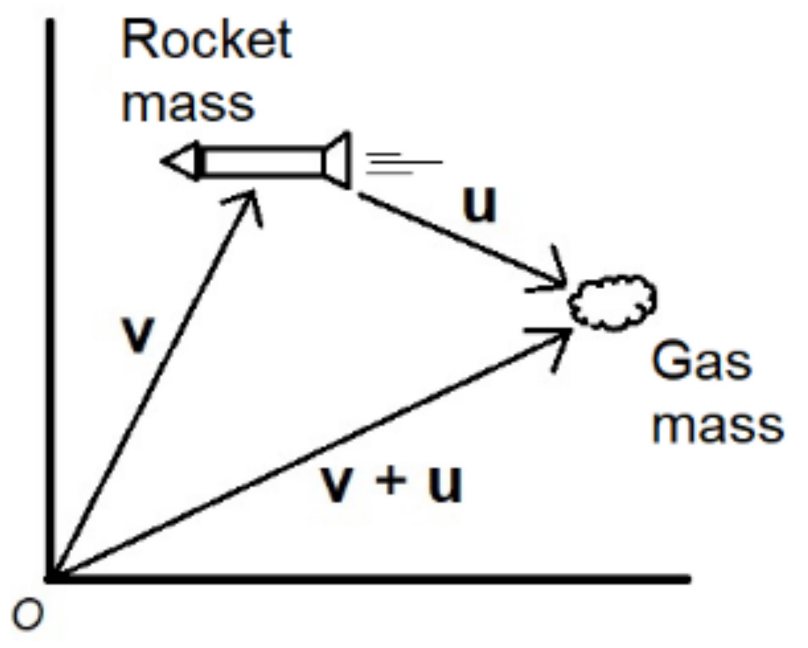

FIGURE 2. Rocket ejecting gas with velocity $\mathbf{u}[10]$.

the mass and velocity of the rocket at time $t+d t$ is equal $m+d m$ and $\mathbf{v}+d \mathbf{v}$, respectively, where $d m=-|d m|$ is a small decrease in rocket mass due to its loss of fuel in the short time $d t$. The gas mass ejected is then $-d m$ and has velocity $\mathbf{v}+\mathbf{u}$ relative to the ground, as shown in Fig. 2 .

The total linear momentum of the rocket-gas system is

$$
\begin{aligned}
& \mathbf{P}_{T}=(m+d m)(\mathbf{v}+d \mathbf{v})-d m(\mathbf{v}+\mathbf{u}) \\
& \mathbf{P}_{T}=m \mathbf{v}+m d \mathbf{v}-\mathbf{u} d m .
\end{aligned}
$$

In Eq. (4), the small term $d m d \mathbf{v}$ has been neglected. The net force over the system, by the second law of Newton, is

$$
\begin{aligned}
& \mathbf{F}=\lim _{x \rightarrow 0} \frac{\Delta \mathbf{P}}{\Delta t}=\lim _{x \rightarrow 0} \frac{\Delta \mathbf{P}_{f}-\Delta \mathbf{P}_{i}}{\Delta t}, \\
& \text { or } \quad m \frac{d \mathbf{v}}{d t}=\mathbf{u} \frac{d m}{d t}+\mathbf{F} .
\end{aligned}
$$

Equation (5) is known as the rocket equation.

\section{Velocity as a function of time}

In this section, two different situations can be considered, one in which the rocket is leaving the Earth's surface and other in which the rocket has already left the Earth's surface and finds itself in outer space without any external forces acting on it.

\section{Free Space}

Assuming a constant gas-expelled velocity in free space, there are no external forces acting on the rocket and the force $\mathbf{F}=0$. From Eq. (5), using $\mathbf{v}=v \hat{\jmath}$ and $\mathbf{u}=-u \hat{\jmath}$, where $u$ is constant, we get

$$
m \frac{d v}{d t}=-\left(\frac{d m}{d t}\right) u
$$


The right term in Eq. (6) is known as the thrust; it acts to propel the rocket forward and it is a negative quantity. That differential equation can be solved to yield

$$
\int_{v_{0}}^{v} d v=-u \int_{m_{0}}^{m} \frac{d m}{m},
$$

where $m$ is the mass of the rocket's structure and the engine, $m_{0}$, is the initial fuel plus $m$. Thus, we have the velocity equation as a function of the relation between the fuel and useful load

$$
\begin{aligned}
v & =v_{0}-u \ln \left(\frac{m}{m_{0}}\right), \\
\text { or } \quad v & =v_{0}+u \ln \left(\frac{m}{m_{0}}\right) .
\end{aligned}
$$

This equation implies that a large amount of fuel is needed $\left(m_{0} / m>1\right)$ to reach high velocities.

\section{Air resistance}

The function $f(v)$ that gives the magnitude of the air resistance varies with speed $v$ in a complicated way. It is quite useful to describe it with a Taylor expansion series as

$$
f(v)=a+b v+c v^{2}+\ldots,
$$

in which the term $a=0$ when $f=0$ with a speed $v=0$. This implies both linear and quadratic terms for the function $f(v)$. That is

$$
f(v)=f_{\text {lin }}+f_{\text {quad }},
$$

where $f_{\text {lin }}$ and $f_{\text {quad }}$ stand for the linear and quadratic terms, respectively. There are objects in which the linear term dominates over the function and the quadratic term can be discarded; this happens in large objects inside highly viscous fluids, like a sphere that moves through dense honey. The linear term arises from the viscous drag of the medium and is generally proportional to the viscosity of the medium and linear size of the projectile.

On the other side, for most objects that move like projectiles, such as tennis balls, bowling balls, and even the human body, the dominant term in the air resistance force is quadratic and the linear term can be discarded. The quadratic term is proportional to the density of the medium and the cross-sectional area of the projectile.

\section{The model problem}

A system composed of three parts is introduced in this section: a mobile that slides without friction over a smooth surface at a certain height; a spring built into the mobile, which is fully compressed at the initial time; and an idealized rocket that acts as a point mass laying on the mobile beside the spring (Fig. 3).

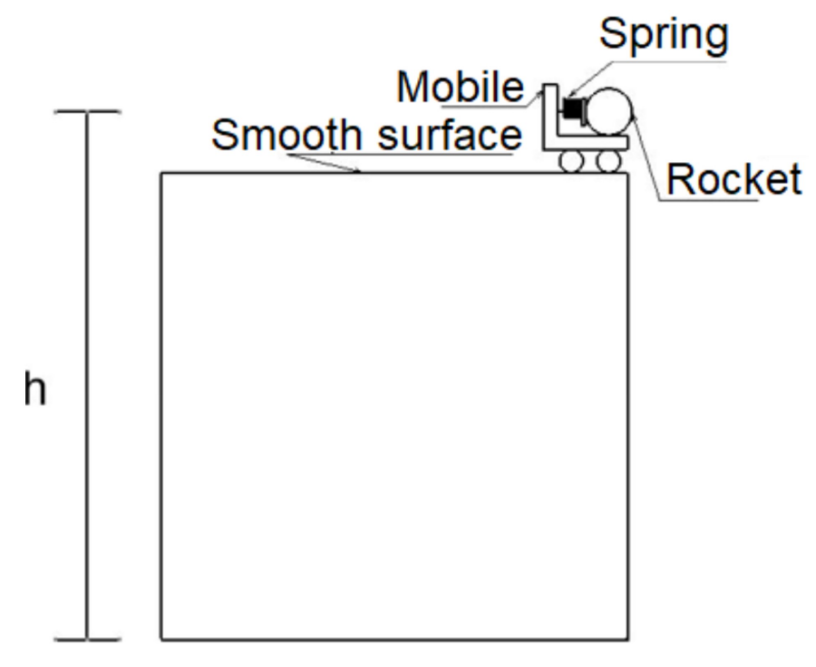

FIGURE 3. Setup sketch.

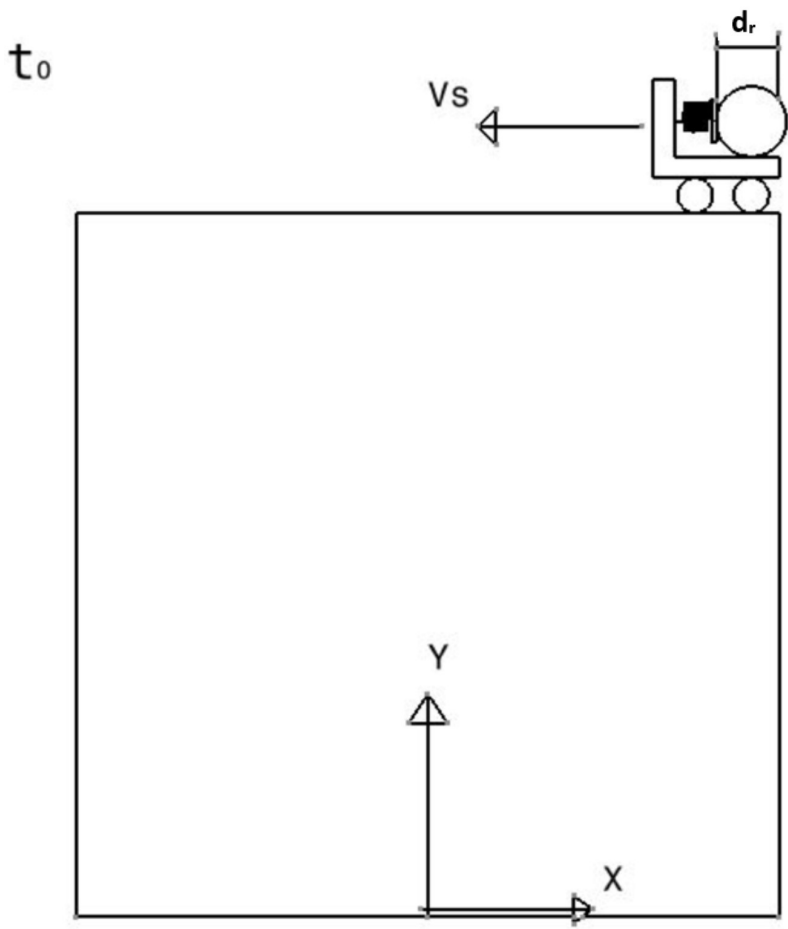

FiguRE 4. System at time $t_{0}$.

At time $t_{0}$, the system has a constant inertial velocity $v_{s}$, as shown in Fig. 4.

At time $t_{1}$, the spring and the rocket are set free in such a way that at time $t_{2}$, the rocket reaches a null inertial velocity, as shown in Fig. 5. This way, at time $t_{2}$, the gravitational effects will cause a free-fall motion on the rocket.

Now, if the effects of the spring at the time when it is released are viewed from a local reference frame fixed in the mobile, and assuming that the idealized rocket slides without friction over the mobile, the following equations describe the motion of the rocket from a non-inertial reference frame: 


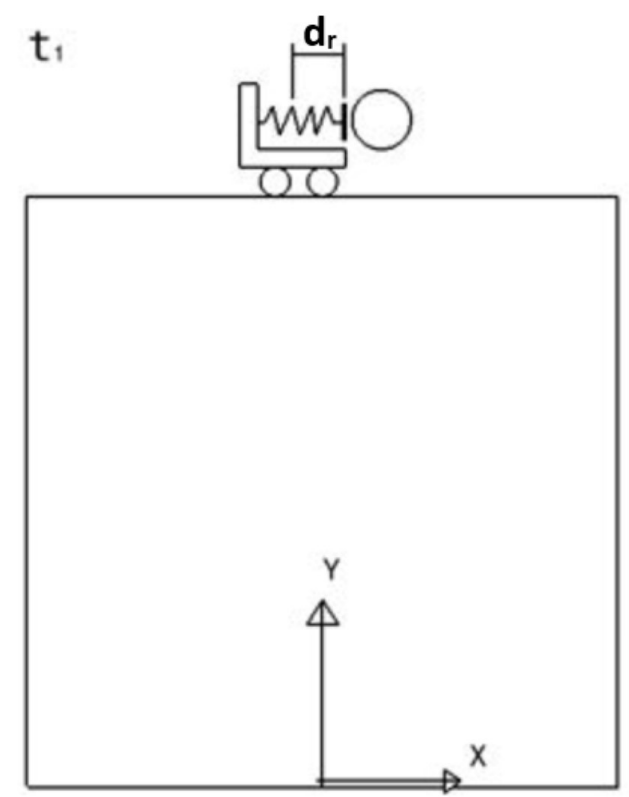

FIGURE 5. System at time $t_{1}$ and $t_{2}$.

$$
\begin{aligned}
& \sum F_{c x}=m_{c} a_{c x}, \\
& -K x(t)=m_{c} a_{c x},
\end{aligned}
$$

where: $a_{c x}=\ddot{x}(t)$, so:

$$
m_{c} \ddot{x}(t)+K x(t)=0 .
$$

As the system is being observed from a non-inertial reference frame, the velocity $v_{C L 0}=0$; also, the initial distance $x_{o}$ is equal to the compression distance of the spring $d_{r}$.

$$
x_{0}=-d_{r} \quad v_{C L 0}=0 \quad \frac{\mathrm{m}}{\mathrm{s}}
$$

Solving the differential equation (13) under the initial conditions allows us to obtain the equation for relative horizontal displacement (14)

$$
x(t)=-d_{r} \cos (\omega t),
$$

where: $\omega=\sqrt{K / m_{c}}$. To obtain velocity $v_{C L}(t)$, is necessary to differentiate Eq. (14)

$$
v_{C L}(t)=\dot{x}(t)=\omega d_{r} \sin (\omega t) .
$$

For the inertial horizontal velocity to be null at time $t_{2}$, condition Eq. (16) must be satisfied.

$$
v_{C L 2}\left(t_{2}\right)=v_{C L 2}=-v_{s}
$$

If the inertial reference frame $v_{s}$ is negative, condition (16) shows that the relative velocity of the rocket has reached the same magnitude as the inertial velocity of the system and in the opposite direction. In that instant, the rocket will start a free fall in which, if no external horizontal forces act on the rocket, it would fall in a straight line until it reaches the ground.
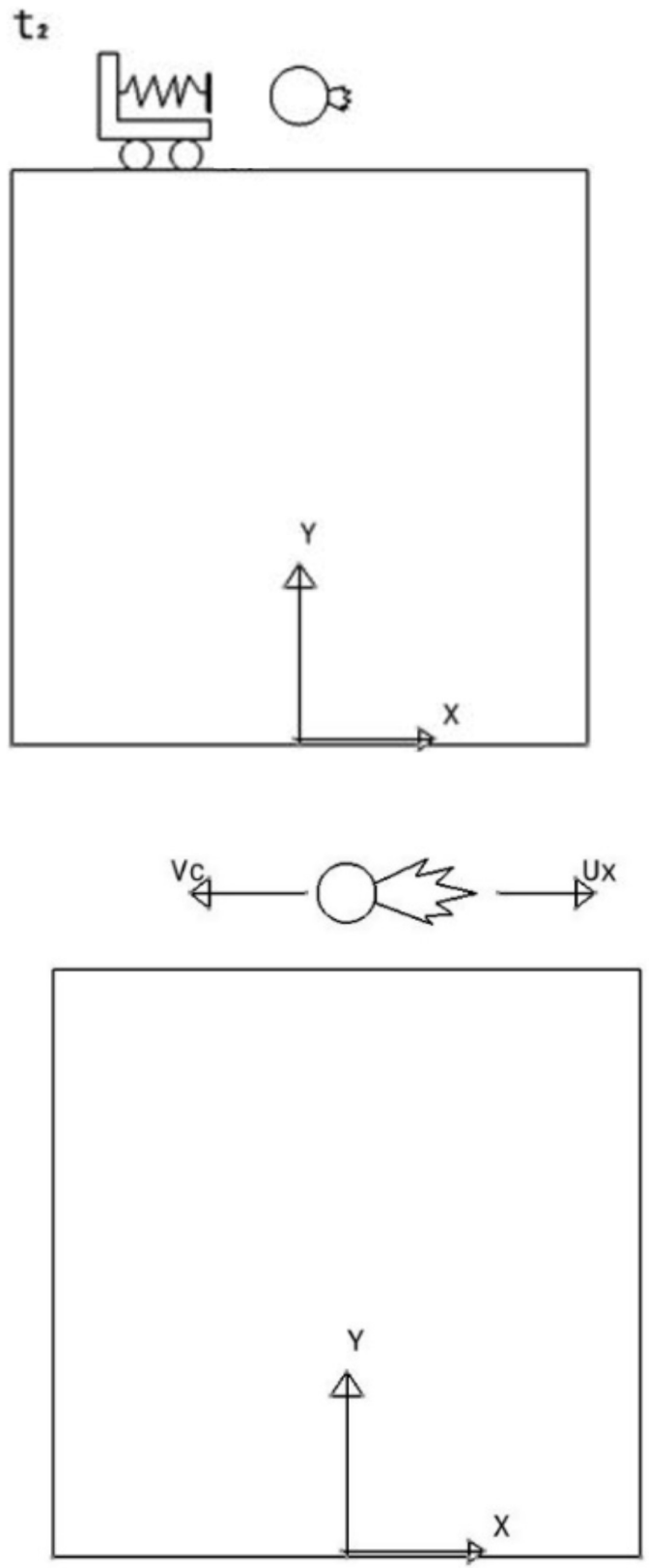

FIGURE 6. Sketch of the rocket at time $t_{2}$.

To analyze the horizontal deviation of the rocket from its free-fall trajectory because of the external force produced by the expulsion of a gas contained inside the rocket, we assume that the rocket should have a deviation in the opposite direction of the freed gas velocity. Figure 6 shows a sketch of the rocket at time $t_{2}$, exactly when the gas is released. Assuming a constant gas-expelled velocity $\mathrm{u}$, the following equations describe the motion of the rocket from time $t_{2}$.

$$
\begin{aligned}
& \sum F_{c y}=m_{c} \frac{d v_{c y}}{d t}-u_{y} \frac{d m}{d t} \\
& \sum F_{c x}=m_{c} \frac{d v_{c x}}{d t}-u_{x} \frac{d m}{d t}
\end{aligned}
$$

where: $u_{x} \gg u_{y}$, so $u_{y} \cong 0 \mathrm{~m} / \mathrm{s}$. 
From the vertical forces balance, Eq. (17), we have

$$
\begin{aligned}
\sum F_{c y} & =m_{c} \frac{d v_{c y}}{d t}=m_{c} a_{c y}, \\
a_{c y} & =-g, \\
-m_{c} g & =m_{c} \frac{d v_{c y}}{d t}+u_{y} \frac{d m}{d t} .
\end{aligned}
$$

From the vertical forces balance, Eq. (18), two cases can be derived.

\section{Case I: No air resistance}

In this case, there are no external forces in the horizontal direction acting on the rocket other than the one generated by the expulsion of the gas.

$$
0=m_{c} \frac{d v_{c x}}{d t}+u_{x} \frac{d m}{d t}
$$

Solving Eq. (22), an expression for the horizontal velocity of the rocket as a function of time can be obtained.

$$
\begin{aligned}
m_{c} \frac{d v_{c x}}{d t} & =u_{x} \frac{d m}{d t} \\
\frac{d v_{c x}}{d t} & =-\frac{u_{x}}{m_{c}} \frac{d m}{d t} \\
d v_{c x} & =-u_{x} \frac{d m}{m_{c}} \\
\int_{v_{c x i=0}}^{v_{c x f}} d v_{c x} & =-u_{x} \int_{m_{c i}}^{m_{c f}} \frac{d m}{m_{c}} \\
v_{c x f} & =-u_{x} \ln \frac{m_{c f}}{m_{c i}}
\end{aligned}
$$

where $v_{c x f}=v_{c x}(t)$,

$$
m_{A}=\rho_{A} A u_{x},
$$

where $\rho_{A}$ denotes the expelled gas density, $A$ is the crosssectional area where the gas is being expelled and $u_{x}=$ const.

$$
m_{c f}=m_{c}(t)=m_{c i}-\rho_{A} A u_{x} t,
$$

so

$$
\begin{aligned}
& v_{c x}(t)=-u_{x} \ln \left(\frac{m_{c i}-\rho_{A} A u_{x} t}{m_{c i}}\right), \\
& v_{c x}(t)=-u_{x} \ln \left(1-\frac{\rho_{A} A u_{x} t}{m_{c i}}\right) .
\end{aligned}
$$

By integrating Eq. (31), we obtain the deviation of the rocket due to of the expelled gases as a function of time.

$$
\begin{aligned}
x_{c}(t) & =-u_{x} \ldots\left\{\frac{\rho_{A} A u_{x} t-m_{c i}}{\rho_{A} A u_{x}}\left[\ln \left(1-\frac{\rho_{A} A u_{x} t}{m_{c i}}-1\right)\right]\right. \\
& \left.+\frac{\rho_{A} A u_{x} t-m_{c i}}{\rho_{A} A u_{x}}\right\}
\end{aligned}
$$

In the vertical direction, the only external force present is the one applied by the gravitational field of the Earth, which leads to Eq. (33).

$$
-m_{c} g=m_{c} \frac{d v_{c y}}{d t}+u_{y} \frac{d m}{d t},
$$

where: $u_{y}=0 \mathrm{~m} / \mathrm{s}$ y $v_{c y i}=0 \mathrm{~m} / \mathrm{s}$

$$
\begin{aligned}
-m_{c} g & =m_{c} \frac{d v_{c y}}{d t} \\
d v_{c y} & =-g d t \\
v_{c y}(t) & =-g t
\end{aligned}
$$

so, by integrating Eq. (36)

$$
y_{c}(t)=\frac{g}{2} t^{2}-y_{c i} .
$$

Equation (37) and its equivalent expressions for all different cases are essential because by solving them for time $t$ in which $y_{c}(t)=0$, a time limit for the rocket to develop a deviation from its free-fall trajectory is determined.

\section{Case II: Considering linear air resistance.}

Since the study is stated for an idealized rocket as a particle with small dimensions, low velocities, and low heights, this case will only consider a linear air resistance.

$$
\begin{gathered}
F_{d x}=m_{c} \frac{d v_{c x}}{d t}+\frac{d m}{d t} \\
m_{c} \frac{d v_{c x}}{d t}+u_{x} \frac{d m}{d t}=-b v_{c x},
\end{gathered}
$$

where $F_{d x}=b v_{c x}$ (linear term). In particular, for a spherical projectile, the coefficient $b$ have the form $b=\beta D v_{c x}$, where $D$ denotes the diameter of a sphere and the coefficient $\beta$ depend on the nature of the medium $\left(\beta=1.6 \times 10^{-4} \mathrm{~m} / \mathrm{s}\right.$ in air at standard temperature and pressure).

In addition, as the changes of mass and velocity are dependent on time, by applying the chain rule, these changes can be expressed with respect to a mass deferential instead of a time deferential, giving us

$$
\frac{d}{d t}=\frac{d m}{d t} \frac{d}{d m}=\alpha \frac{d}{d m},
$$

whit

$$
\begin{aligned}
\alpha \frac{d}{d m} & =\frac{d}{d t}, \\
\alpha & =\frac{d m}{d t} .
\end{aligned}
$$

The coefficient $\alpha$ represents the mass flow rate. Thus, Eq. (39) can be expressed as

$$
\begin{aligned}
m_{c} \alpha \frac{d v_{c x}}{d m}+\alpha u_{x}+b v_{c x} & =0 \\
\frac{d v_{c x}}{-b v_{c x}-\alpha u_{x}} & =\frac{d m}{m_{c \alpha}}
\end{aligned}
$$




$$
\begin{gathered}
\int_{0}^{v_{c x}} \frac{d v_{c x}}{b v_{c x}+\alpha u_{x}}=-\int_{m_{c i}}^{m_{c f}} \frac{d m}{m_{c \alpha}} \\
\frac{1}{b}\left(\operatorname{Ln}\left|b v_{c x}+\alpha u_{x}\right|-\operatorname{Ln}\left|\alpha u_{x}\right|\right)=\frac{1}{\alpha}\left(\operatorname{Ln}\left(m_{c f}\right)\right. \\
\left.-\operatorname{Ln}\left(m_{c i}\right)\right) \\
\operatorname{Ln}\left(1+\frac{b v_{c x}}{\alpha u_{x}}\right)=\frac{b}{\alpha} \operatorname{Ln}\left(\frac{m_{c f}}{m_{c i}}\right) \\
1+\frac{b v_{c x}}{\alpha u_{x}}=\left(\frac{m_{c f}}{m_{c i}}\right)^{\frac{b}{\alpha}} \\
v_{c x}(t)=\frac{\alpha u_{x}}{b}\left(\left(\frac{m_{c f}}{m_{c i}}\right)^{\frac{b}{\alpha}}-1\right) .
\end{gathered}
$$

From Eq. (42), we have

$$
\alpha=-\rho_{A} A u_{x}
$$

So, Eq. (49) takes the form

$$
v_{c x}(t)=\frac{-\rho_{A} A u_{x}^{2}}{b}\left(\left(\frac{m_{c f}}{m_{c i}}\right)^{\frac{-b}{\rho_{A}^{A} u_{x}}}-1\right) .
$$

Note that, $m_{c f}=m_{c i}-\rho_{A} A u_{x} t$,

$$
\begin{aligned}
& v_{c x}(t)=\frac{-\rho_{A} A u_{x}^{2}}{b}\left(\left(\frac{m_{c i}-\rho_{A} A u_{x} t}{m_{c i}}\right)^{\frac{-b}{\rho_{A} A u_{x}}}-1\right), \\
& v_{c x}(t)=\frac{-\rho_{A} A u_{x}^{2}}{b}\left(\left(1-\frac{\rho_{A} A u_{x} t}{m_{c i}}\right)^{\frac{-b}{\rho_{A} A u_{x}}}-1\right) .
\end{aligned}
$$

By integrating Eq. (53), we obtain the deviation of the rocket due to the effects of the expelled gases as function of time and while considering air resistance.

$$
\begin{aligned}
x_{c}(t) & =-\frac{-\rho_{A} A u_{x}^{2}}{b} \ldots\left(\frac{\left(m_{c} i-\rho_{A} A u_{x} t\right)}{b-\rho_{A} A u_{x}}\right. \\
& \left.\times\left(1-\frac{\rho_{A} A u_{x} t}{m_{c i}}\right)^{\frac{-b}{\rho_{A} u_{x}}}-t-\frac{m_{c i}}{b-\rho_{A} A u_{x}}\right)
\end{aligned}
$$

As for the vertical direction, because the gas is being expelled horizontally, the equations for velocity and position will be the following:

$$
-b v_{c y}-m_{c} g=m_{c} \frac{d v_{c y}}{d t}+u_{y} \frac{d m}{d t},
$$

where: $u_{y}=0 \mathrm{~m} / \mathrm{s}$ and $v_{c y i}=0 \mathrm{~m} / \mathrm{s}$.

Also, as the mass of the expelled gas much smaller than the initial mass of the system, we can consider the mass of the system to be constant along the displacement on the vertical direction or even that the air resistance can be neglected; this second assumption can be made because the small velocities and reduced dimensions of the stated system.
Case II.1: Constant mass of the system

$$
\begin{aligned}
& -b v_{c y}-m_{c} g=m_{c} \frac{d v_{c y}}{d t} \\
& \frac{d v_{c y}}{d t}+\frac{b}{m_{c}} v_{c y}+g=0 \\
& v_{c y}(t)=\frac{g m_{c}}{b}\left(e^{\frac{-b t}{m_{c}}}-1\right) \\
& y_{c}(t)=\frac{g m_{c}}{b}\left(-\frac{m_{c} e^{\frac{-b t}{m_{c}}}}{b}-t+\frac{m_{c}}{b}\right)+y_{c i}
\end{aligned}
$$

\section{Case II.2: Neglectable air resistance in the vertical direc-} tion.

$$
\begin{aligned}
-m_{c} g & =m_{c} \frac{d v_{c y}}{d t} \\
-g & =\frac{d v_{c y}}{d t} \\
v_{c y}(t) & =g t \\
y_{c}(t) & =\frac{g}{2} t^{2}-y_{c i}
\end{aligned}
$$

From cases II.1 and II.2, a discrepancy between both considerations will be observed.

\section{Cases study}

After developing the fundamental equations under the previously stated conditions, the physics problem is fully defined. At this point, it is possible to assign values to fixed parameters, such as the rocket mass $m_{c}$, aperture area of the rocket A from where the gas is expelled, gravity magnitude $\mathrm{g}$, the expelled gas density (air) $\rho_{A}$, idealized rocket diameter $D$, and linear air resistance constant $\beta$. All these parameters are listed in Table I.

TABLE I. Parameters of the case study.

\begin{tabular}{ll}
\hline Parameter & Unit \\
\hline$m_{c}$ & $0.5 \mathrm{~kg}$ \\
$\mathrm{~A}$ & $3.1416 \times 10^{-6} \mathrm{~m}^{2}$ \\
$\mathrm{~g}$ & $9.81 \mathrm{~m} / \mathrm{s}^{2}$ \\
$\rho_{A}$ & $1.225 \mathrm{~kg} / \mathrm{m}^{3}$ \\
$D$ & $0.06 \mathrm{~m}$ \\
$\beta$ & $1.6 \times 10^{-4} \mathrm{Ns} / \mathrm{m}^{2}$ \\
$b$ & $9.6 \times 10^{-6} \mathrm{~kg} / \mathrm{s}$ \\
\hline
\end{tabular}




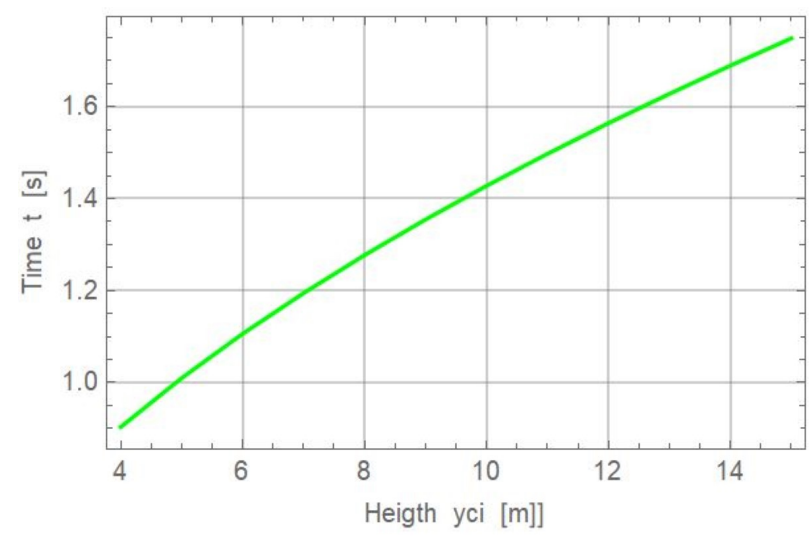

FIGURE 7. Free fall time case I.

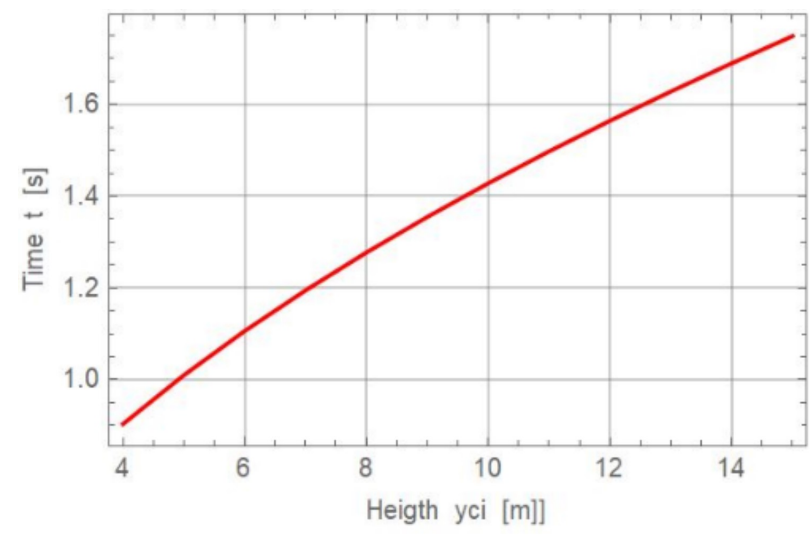

FIGURE 8. Free fall time case II.1.

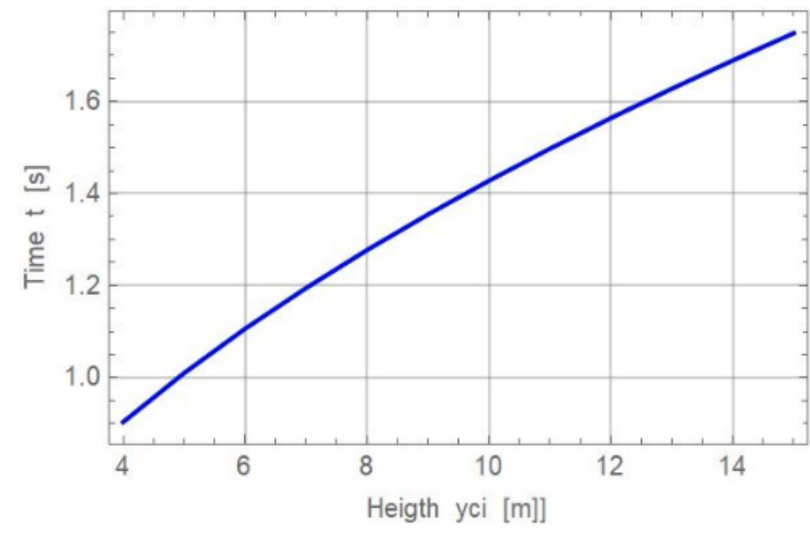

FIGURE 9. Free fall time case II.2.

Now, it is possible to use Eqs. (27), (49), and (53) to obtain the time that takes the rocket to reach the ground from the instant when it is released. For evaluating purposes, it's necessary to variate the initial height value $y_{c i}$ within a certain range applicable for all cases: $4 \leq y_{c i} \leq 15 \mathrm{~m}$.

Figures 7, 8, and 9 represent the free-fall times as a function of initial height $y_{c i}$ for cases I, II.1, and, II.2, respectively.

Note that Eqs. (27) and (53) are the same, so the free-fall times for cases I and II.2 are the same. Likewise, the average

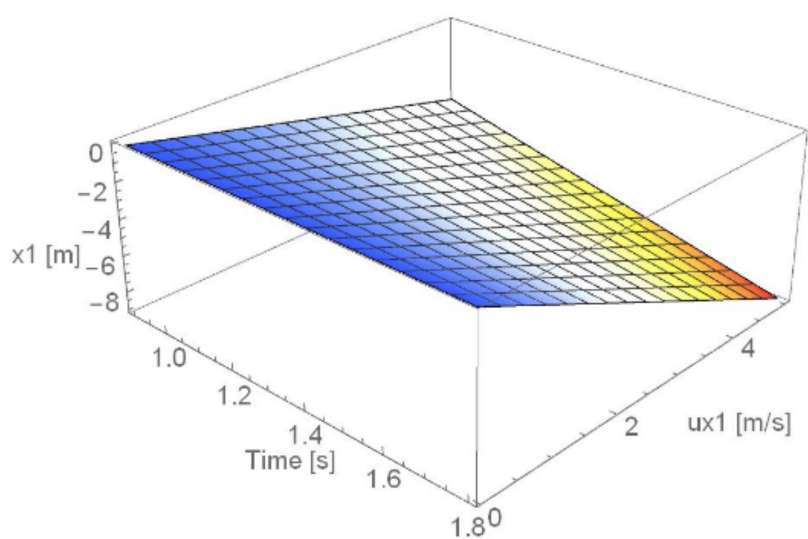

FIGURE 10. Horizontal deviation as function of time and gas velocity, Case I.

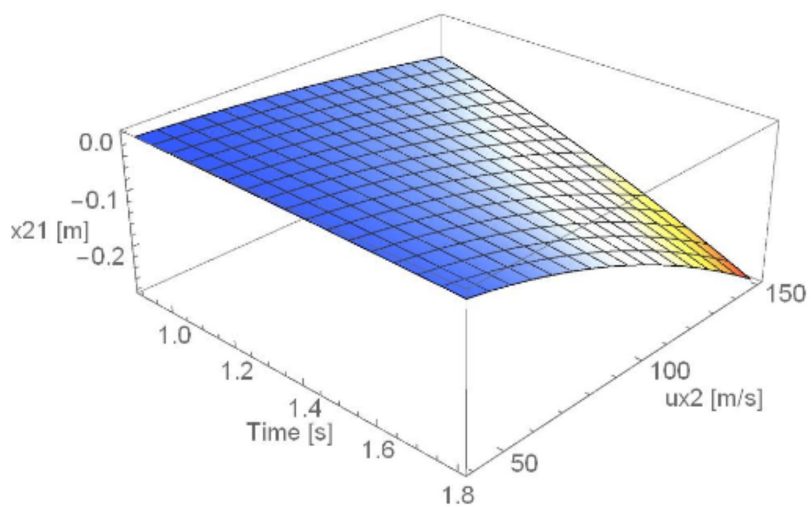

FIGURE 11. Horizontal deviation as function of time and gas velocity, Case II. 1 and II.2.

difference between the free fall times of cases I and II.1 is $5.8 \times 10^{-6} \mathrm{~s}$, and therefore, they can be considered equal.

Finally, in order to obtain the horizontal deviation of the rocket, the values of expelled gas velocity values can vary when using the time range $0.90304 \leq t \leq 1.74874 \mathrm{~s}$. It is necessary to remark that the velocity ranges for cases II. 1 and II. 2 have larger magnitudes due to the air resistance; knowing this, the expelled gas velocity ranges are:

Case I: $50 \times 10^{-3} \leq u_{x} \leq 1.74874 \mathrm{~m} / \mathrm{s}$.

Cases II.1 and II.2: $400 \times 10^{-1} \leq u_{x} \leq 150 \mathrm{~m} / \mathrm{s}$.

\section{Results}

Figure 10 contains the plot for the horizontal rocket deviation as a function of time and expelled gas velocity for case I using Eq. (22). For the horizontal rocket deviations of cases II.1 and II.2. Figure 11 contains the same graph, because both cases use Eq. (44).

Figure 12 shows a graph of the trajectories of the rocket where the green line represents free-fall motion with $u_{x}=0$ $\mathrm{m} / \mathrm{s}$. The red line represents case I where the air resistance is neglected and an expelled gas velocity of $u_{x}=50 \times 10^{-3} \mathrm{~m} / \mathrm{s}$ 


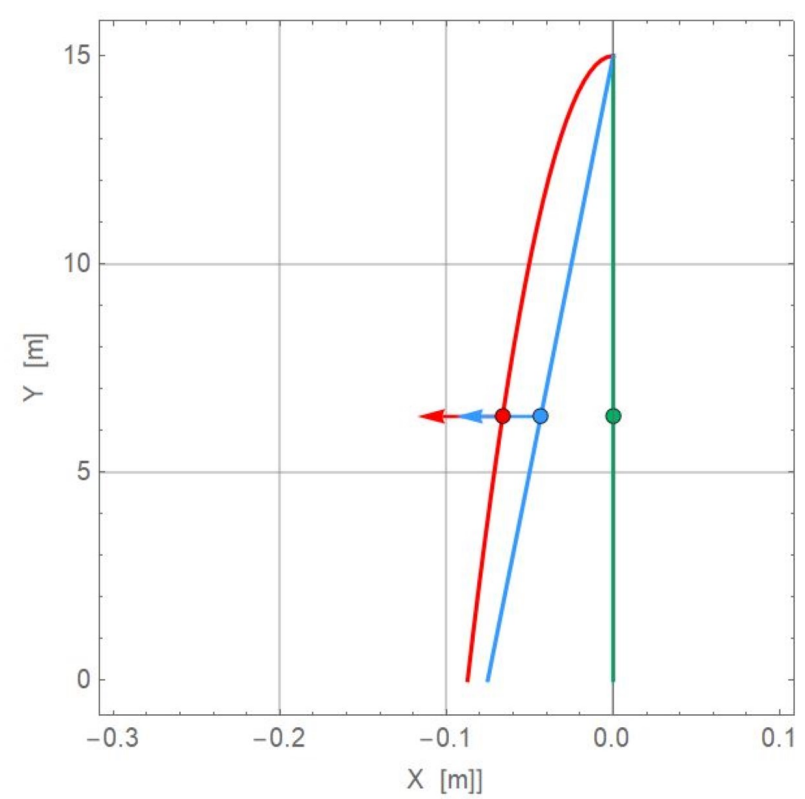

FIGURE 12. Rocket trajectories, Free fall (Green), Case I (Red), Case II (Blue).

is used. The blue line represents cases II.1 and II.2 in which linear air resistances are considered and an expelled gas velocity of $u_{x}=800 \times 10^{-1} \mathrm{~m} / \mathrm{s}$ is used; this large magnitude of expelled gas velocity for case II was arbitrarily selected to have the graphs on a similar scale. For all cases, a height of $15 \mathrm{~m}$ and a respective time interval of: $0 \leq t \leq 1.74874 \mathrm{~s}$ are used.

\section{Discussion}

In both case studies, the horizontal deviation due to the expulsion of the gas is more noticeable when the gas velocity or the time increases. However, in these case studies, we decided to use air as the gas was expelled and to fix a value for the mass and diameter of the idealized rocket. All these parameters could also be varied to increase the horizontal deviation.

In the case studies, external forces are considered to act on the center of gravity of the rocket in purely horizontal or vertical directions so that they do not generate any moment on the rocket's center of gravity.
In practice, different difficulties would be presented when one tried to carry out the experiment, such as determining the time in which the spring is released to ensure that the inertial horizontal velocity of the rocket is zero, determining a geometry for the idealized rocket that allows it to maintain its orientation without turning on its center of gravity, or implementing a system that keeps the expelled gas velocity effectively constant.

On the other hand, the proper experiment requires the measurement and determination of other necessary physical parameters that are not within the scope of this work, e.g., the friction forces between sliding elements, dimensions of the mobile, elastic spring constant, dimensions of the rocket, materials to be used, volume of the gas, and pressure level.

In addition, if the experiment is performed in a vacuum, the condition of zero air resistance can be ensured, thus presenting an area of opportunity for future work that gives continuity to this topic.

\section{Conclusions}

In this work, the equations of motion to cancel the inertial velocity of a rocket mounted on a mobile that moves at a constant inertial velocity have been formulated. It was done through the application of an elongation force of a spring coupled to the mobile, which causes the relative speed of the rocket on the mobile.

In this way, both for the case that does not consider the air resistance as well as for the case that considers the air resistance, under the conditions described in this work, it was possible to formulate the equations of movement of the rocket from the moment in which its inertial speed is zero and a free-fall movement begins. Through these equations it can be verified that for vertical movement when there is no external force that drives the rocket in that direction, the effects of air resistance are negligible.

Finally, it was observed that for a rocket that expels a gas as a method of propulsion, several parameters, such as the velocity of the gas, the mass of the system, the time during which the gas is expelled, the geometry of the rocket, and the coefficient of resistance of the air, significantly impact the velocity and displacement of the rocket.
1. I. Newton, Philosophiae Naturalis Principia Mathematica, 3rd ed. (London, Apud Guil and Joh Innys, 1726).

2. P. Timmerman and J. P. van der Weele, On the rise and fall of a ball with linear or quadratic drag, Am. J. Phys. 67 (1999) 538, https://doi.org/10.1119/1.19320

3. H. Rodrigues, M. O. de Pinho, D. Portes Jr., and A. Santiago, Analytical description of ascending motion of rockets in the atmosphere, Eur. J. Phys. 30 (2009) 185, https: / / doi .org/ $10.1088 / 0143-0807 / 30 / 1 / 019$

4. V. Ivchenko, On projectile motion with quadratic drag force,
Eur. J. Phys. 39 (2018) 045004, https://doi.org/10. 1088/1361-6404/aab343

5. C. Siebert, P. R. DeStefano, and R. Widenhorn, Comparative modeling of free fall and drag-enhanced motion in the classical physics drop experiments, Eur. J. Phys. 40 (2019) 045004, https://doi.org/10.1088/1361-6404/ab1fbc

6. A. V. Kraff, G. S. Vázquez, R. R. Mijangos, and J. A. HerediaCancino, Obtención y solución a la ecuación de movimiento de un cohete, actuando sobre él las fuerzas externas del campo gravitacional constante y el rozamiento del aire proporcional a 
la velocidad, Rev. Mex. Fis. E 61 (2015) 6, https://rmf. smf.mx/ojs

7. H. E. Caicedo-Ortíz, E. Santiago-Cortés, H. O. Castañeda Fernandez, and C. Hernández Hernández, Cohetes hidráulicos con videos en cámara lenta, Rev. Mex. Fis. E 64 (2018) 232, https://doi.org/10.31349/RevMexFisE.64. 232

8. S. M. Stewart, An analytic approach to projectile mo- tion in a linear resisting medium, Int. J. Math. Educ. Sci.

Technol. 37 (2006) 411, https://doi.org/10.1080/ 00207390600594911

9. D. A. Morales, A generalization on projectile motion with linear resistance, Can. J. Phys. 89 (2011) 1233, https: / / doi. org/10.1139/p11-121

10. J. E. Hasbun, Classical mechanics with MATLAB applications (Jones and Barlett, Sudbury, 2009). 